\title{
\$ LA UNIVERSIDAD EN UN HORIZONTE DE PERPLEJIDAD
}

Iván Carvajal

ivcarvaj@gmail.com

\section{Resumen}

El debate en torno de lo que podría ser la «misión de la universidad» en el Ecuador requiere de una actitud (un êthos filosófico) semejante a la que propone Foucault en Qu'est-ce que les Lumières?, que coloca como tarea la comprensión de la actualidad. El ensayo retoma la crítica del pensamiento universitario ecuatoriano, especialmente de La universidad, sede de la razón de Hernán Malo González, a fin de señalar a la vez la importancia y continuidad de la defensa de la racionalidad, la autonomía y la apelación al diálogo, y su insuficiencia para comprender la actualidad. Esta no puede ser comprendida desde una visión circunscrita al Estado nacional o desde la «ecuatorianización» de la universidad, sino a partir de una apertura a los problemas globales que afectan al conjunto de la humanidad, ante todo el ecocidio (y dentro de este, la crisis del cambio climático), y la posibilidad cierta de extinción de lo humano o de su trasmutación (lo poshumano).
A la vez, es necesario comprender la crisis política marcada por la declinación de los Estados nacionales y de la democracia (liberal-representativa o directa-plebiscitaria). La actualidad se presenta como un horizonte de perplejidad. Para comprender la actualidad es preciso apelar a un «êthos filosófico» que posibilite la apertura de unas nuevas Humanidades, que tendrían que abrirse paso en la universidad en permanente contradicción con la ideología tecnocrática dominante en ella y con los poderes (políticos, corporativos, religiosos...) que actúan sobre la universidad. La emergencia de unas nuevas Humanidades, que intentan configurarse en el presente, requiere a su vez de una universidad abierta.

Palabras clave: universidad, pensamiento ecuatoriano, Humanidades, ecocidio, actualidad. 


\title{
THE UNIVERSITY ON A HORIZON OF PERPLEXITY
}

\begin{abstract}
The debate about what the "mission of the university" in Ecuador could be, requires an attitude (a philosophical êthos) similar to the one proposed by Foucault in Qu'est-ce que les Lumières?; which places as a task the understanding of the present. This essay takes up the criticism of Ecuadorian university thought, especially of The University, core of Hernán Malo González's reason, in order to point out both the importance and the continuity of the defense of reasonableness, autonomy; the call for dialogue, and its insufficiency to understand the present. This cannot be understood from a vision limited to the Nation-State or from the "Ecuadorianization" of the university, but from an openness to the global problems that affect the whole of humanity, above all ecocide (and within it, the crisis of climate change), and the real possibility of human being's extinction or of their transmutation (the post-humanism). At the same time, it is necessary to understand the political crisis marked by the decline of the Nation-States and of democracy (liberal-representative or direct-plebiscitary). The present time is presented as a horizon of perplexity. To understand the present, it is necessary to invoke to a "philosophical êthos" that makes possible space for new Humanities, which would have to make their way into the university in permanent contradiction with the technocratic ideology that dominates in it, and with the powers (political, corporate, religious ...) that act on the university. The development of new Humanities, which are attempting to shape themselves in the present, requires an open university.
\end{abstract}

Key words: university, Ecuadorian thought, humanities, ecocide, present. 
Michel Foucault se ocupó del problemático legado de la llustración (les Lumières) en uno de sus últimos trabajos (1984), en el que comenta el ensayo que, un par de siglos antes, había dedicado Kant para responder a la pregunta propuesta por el periódico Berlinische Monatschrift, «QQué es la Ilustración? ( «Was ist Aufklärung?»). A Foucault le interesa ante todo la concepción del presente y de la historia que Kant expone en ese ensayo, de ahí que la contraste con aquellas que habían expuesto anteriormente Mendelssohn y Vico. Luego contrapone la idea kantiana de llustración a la idea de modernidad que plantearía Baudelaire medio siglo más tarde, y más adelante anota la diferencia entre humanismo e llustración. El comentario de Foucault se dirige a situar una posición crítica sobre la llustración desde la actualidad, que no se reduzca a la mera adopción de sus legados ni tampoco los rechace sin más; de ahí la importancia que otorga a la concepción del presente, es decir, de la actualidad en Kant. Lo que por mi parte quisiera resaltar aquí es la cuestión de orden ético que coloca Foucault, o como él la llama, de actitud filosófica (una «attitud limite») en torno a la actualidad, que proviene de Kant: «el enraizamiento en la Aufklärung de un tipo de interrogación filosófica que problematiza a la vez la relación con el presente, el modo de ser histórico y la constitución de sí-mismo como sujeto autónomo». El vínculo que puede enlazarnos con la llustración no tendría que ver propiamente con aspectos doctrinarios, sino con «la reactivación permanente de una actitud, es decir, de un êthos filosófico que se podría caracterizar como crítica permanente de nuestro ser histórico.» Una crítica que, como se señala en otro pasaje del ensayo foucaultiano, si bien se afianza en la racionalidad, no sería ya trascendental ni debería ser meramente negativa, esto es, no debería encaminarse a establecer los límites de lo que se puede conocer, sino que debería ser positiva y establecer los límites que podrían ser atravesados o transgredidos: «Se trata en suma de transformar la crítica ejercida en la forma de la limitación necesaria en una crítica práctica en la forma de un sobrepasar [franchissement] posible.» Ello implica la indagación histórica («enquête historique») de los acontecimientos que «nos han llevado a constituirnos y a reconocernos como sujetos de lo que hacemos, pensamos, decimos», indagación que para Foucault implica la arqueología y la genealogía que había venido proponiendo durante un par de décadas. El êthos filosófico sería entonces «la ontología crítica de nosotros-mismos como una prueba histórico-práctica de los límites que podemos sobrepasar, y por tanto como trabajo de nosotros-mismos sobre nosotros-mismos en tanto que seres libres.» Tal ontología crítica de 
«nosotros-mismos» sería posible a condición de colocarnos en el límite: «Este êthos filosófico puede caracterizarse como una actitud-límite. No se trata de un comportamiento de rechazo. Debemos escapar de la disyuntiva "afuera-adentro"; hay que colocarse en las fronteras. La crítica es, por supuesto, el análisis de los límites y la reflexión sobre ellos», señala el filósofo francés.

Cabe apelar a este êthos filosófico para abordar la cuestión planteada en nuestro debate, aunque este se circunscriba, al menos en lo inmediato, a la consideración de la universidad (ecuatoriana) en su actualidad. Hay una cuestión que subyace en el texto foucaultiano y que está indicada por un deíctico, el pronombre personal nosotros, incluido en el «nosotros-mismos». ¿A quiénes alude ese nosotros? Es evidente que en cada caso nosotros designa un referente que deberá ser señalado. En principio, aunque de manera poco crítica, se podría considerar que, en el texto de Foucault, nosotros-mismos se refiere a los pensadores europeos o, más ampliamente, de Occidente, situados en la posmodernidad o la tardo-modernidad. La actualidad o el presente implican no solamente una demarcación temporal, inserta a su vez en una idea de tiempo, sino también cierta localización o un marco territorial. La pregunta por la llustración, por lo que queda como su legado problemático, tiene que ver con lo político (el uso «público» de la razón o de la racionalidad, la madurez o la autonomía de los sujetos, las libertades de pensamiento, de expresión, o las condiciones de la democracia - al menos, en el sentido de la democracia liberal), con los saberes, los conocimientos, con expectativas vinculadas al afianzamiento de la racionalidad en la convivencia social. Más allá de los cuestionamientos y críticas que se hacen a los efectos de la llustración en América Latina, es indudable que debemos aún hoy interrogarnos sobre su legado. Sin embargo, esta interrogación cabe plantearla en un horizonte problemático complejo, que requiere de la superación (franchissement, para tomar el término que, como hemos visto, usa Foucault) de cualquier forma de localización que distribuya el mundo del presente bajo cortes dicotómicos, a través de los que a menudo se intenta excluirnos de o contraponernos con Occidente, a pesar de la «mundialización», o de trazar líneas divisorias entre norte y sur que llegan a postular supuestas «epistemologías» distintas. Resulta inquietante que tales propuestas emerjan cuando se debe enfrentar la crisis ecológica global que se evidencia, entre otras formas, en el «calentamiento global», es decir, ante una situación en extremo compleja, que exige conocimientos y acciones globales, dado que la crisis involucra al conjunto de la humanidad: la 
de nuestros días, la de los seres humanos que vendrán luego de nosotros, la de los seres vivos que habitan el planeta. La responsabilidad global, por supuesto, se distribuye de manera diferenciada en relación con las configuraciones políticas, económicas, sociales (Estados, corporaciones, clases o grupos, sujetos).

Lo que quisiera colocar como premisa es que la compresión del nosotros involucrado en el êthos filosófico con el que debemos abordar la actualidad, nos sitúa dentro de un horizonte en el cual la necesaria reflexión sobre la singularidad o la especificidad de cierta configuración histórico-social, los «ecuatorianos» en una coyuntura histórica (la del año 2017), requiere necesariamente de la comprensión de la generalidad, es decir, de lo que acontece en el mundo: nuestra actualidad se caracteriza por la mundialización. No se trata propiamente de una relación entre lo particular y lo universal, sino de las relaciones complejas entre lo local y lo global, entre las singularidades y la generalidad.

El nosotros al que se apela desde la Universidad del Azuay al invitarnos a debatir sobre el «pensamiento universitario ecuatoriano», pareciera referirse a todos aquellos que están involucrados «hoy día», de cualquier manera que fuese, en la reflexión sobre lo que se ha venido denominando «Universidad ecuatoriana», o más ampliamente, a quienes en el Ecuador trabajan como profesores o investigadores o estudian en las universidades y escuelas politécnicas, y aun a los gobernantes y políticos que deciden sobre el destino de las instituciones universitarias del Ecuador. Ahora bien, cualquier reflexión sobre la universidad en el Ecuador debería darse más allá de los límites colocados por la que ha sido nuestra «tradición» de pensamiento universitario, para invocar a nuestros indudables antecesores, tradición que podría identificarse con el lema «ecuatorianizar la universidad», acuñado por el filósofo jesuita Hernán Malo González, entonces rector de la PUCE, hace cuatro décadas². Ese lema sintetiza buena parte de los postulados compartidos por los reformistas universitarios entre 1968 y 1978, entre ellos y en primera línea, Manuel Agustín Aguirre y Hernán Malo González: los ideales de una universidad orientada al desarrollo de la cultura nacional, del conocimiento de la realidad nacional, que debía ofrecer alternativas para el desarrollo nacional, esto es, alternativas que cambiasen la estructura social, que tuviesen como objetivo la soberanía nacional, la justicia social y la democracia; una universidad que impul1 Es decir, cuando la actual Universidad del Azuay era una sede de la PUCE. 
sara el conocimiento, y que incluso fuera capaz de generar una (imposible) ciencia nacional (Aguirre). Malo puso otra cuestión en el debate de ese momento: la universidad tenía que afrontar la «crisis de la razón», con lo cual apuntaba a una crítica de la llustración y del racionalismo moderno, crítica que lamentablemente fue apenas esbozada y no tuvo posterior desarrollo. Después de ese debate, en el Ecuador predominó la idea de que la función social de las universidades se reducía a la preparación de profesionales para el «desarrollo», un tanto al vaivén de las ideologías economicistas provenientes tanto del «neoliberalismo» como del «neokeynesianismo»; por consiguiente, el débil debate sobre la cuestión universitaria en estas últimas tres décadas se ha circunscrito a la «calidad», bajo el supuesto de la «pérdida de calidad» ocasionada por la «masificación» estudiantil que tuvo lugar desde inicios de la década de los años 70 del siglo pasado, frente a lo cual había que proponer acciones encaminadas a mejorar la formación profesional. Se ha recurrido a nociones tan vagas como «excelencia», se ha llegado a proponer que se adopten en las universidades los modelos empresariales de planificación o «calidad total». Neoliberales y neokeynesianos, esto es, partidarios de la «reducción del tamaño del Estado» y partidarios del intervencionismo estatal, comparten presupuestos básicos, inherentes a la ideología tecnocrática que subyace en la reducción de la universidad a una fábrica de técnicos y tecnólogos encargados de la administración de las poblaciones, del gobierno, de las empresas, de los procesos productivos, financieros o comerciales. Igualmente, la investigación universitaria debería orientarse, de acuerdo con esa ideología, a la innovación tecnológica, o al menos a la transferencia tecnológica desde los «países avanzados» a los «países en desarrollo». Esta ideología no es «nacional», es global. Por tanto, en el «pensamiento universitario ecuatoriano» se produjo, hace tres décadas, un tránsito desde la propuesta de «ecuatorianizar la universidad» a la ideología tecnocrática, la cual finalmente, hace aproximadamente una década, durante el gobierno de Correa, acabó por imponerse como discurso oficial de la política de Estado. Malo, hasta cierto punto coincidiendo con la distinción que establecía Horkheimer entre razón objetiva y razón subjetiva («Sobre el concepto de razón») o con Marcuse (El hombre unidimensional) en la crítica al racionalismo moderno, proponía un sentido plural de la razón, o mejor aún de la racionalidad o del pensamiento, que no se redujera a la dianoia o razón «matemática» (del conocimiento) sino que comprendiera las distintas posibilidades de sabiduría (incluido el mito), que fuese fundamento de la «universidad humanística», a la que correspon- 
dería «la visión y el estudio del hombre integral». La consecuencia que saca Malo de su distinción es que habría dos tipos de universidad: la humanística (Sedes Sapientiae) y la técnica (Sedes Rationis); su posición se orientaba a que aquella englobase a esta última. Lo que se impuso, contra ese ideal, fue finalmente la racionalidad instrumental orientada, más que al saber matemático (en sentido amplio, es decir: el conocimiento científico), a sus efectos técni$\cos ^{2}$. No obstante, Malo procuraba «salvar a la razón», como anotara su amigo y comentarista Arturo Roig. Ese esfuerzo guarda correspondencia con lo que Malo quiere preservar para la universidad: la razón como sustento de la autonomía, de la búsqueda de la verdad, de la crítica, del diálogo y de una práctica política encaminada a la justicia, abierta a la sociedad, al compromiso con los pobres. Pero es justamente este núcleo de propósitos, vinculados con cierta crítica de la racionalidad ilustrada y moderna, la que ya estaba en crisis en el momento en que Malo enunciaba sus postulados.

No fue posible para Malo, ni para Aguirre, ni para sus contemporáneos, tomar conciencia de los cambios históricos que se estaban operando desde al menos el fin de la Segunda Guerra Mundial. Su comprensión del presente, para decirlo de alguna manera, estuvo marcada por la teoría de la dependencia y, en correspondencia con esta, por el propósito político de liberación y

2 De ahí deriva la subordinación de toda la organización del quehacer universitario a esa finalidad tecnocrática, que se expresa en la burocratización generalizada de las actividades, de la que se quejan hoy día, y con sobrada razón, profesores, investigadores y estudiantes. Por otra parte, los efectos técnicos del conocimiento científico tienen alcances diferenciados en relación con la concentración del capital; las grandes corporaciones transnacionales concentran las innovaciones tecnológicas, sobre todo aquellas que requieren de grandes recursos (financieros y también de «capital humano»). La concentración de la propiedad de patentes y otros componentes de las tecnologías daría lugar a lo que Bolívar Echeverría denominaba «renta tecnológica», designación que él toma, modificando su sentido, de la «renta de la tierra» (Marx), para dar cuenta de una modalidad contemporánea de distribución de plusvalía y de la generación de plusvalía extraordinaria en el conjunto del sistema capitalista mundial. Creer que por decreto se podía constituir un Silicon Valley criollo, expropiando para tal fin $4.000 \mathrm{Ha}$ de tierras agrícolas, como hizo el gobierno de Correa, demuestra una mezcla de arrogancia, de ignorancia y de voluntad de estafa a la sociedad, por el dispendio de recursos que, incluso dentro de la ideología tecnocrática, podían haber sido destinados a fortalecer el sistema universitario ecuatoriano. Yachay es el emblema de semejante despropósito, así como el satélite Pegaso lo es de la «idea» de progreso científico que imperó durante la década correísta. (Para una crítica consistente de esa política y su ideología, ver A. Villavicencio: 2013, 2016). 
desarrollo nacional. La idea de desarrollo, por lo demás, era compartida por intelectuales ubicados en distintas posiciones políticas, en un haz que iba desde sectores reformistas de la derecha liberal a los revolucionarios de izquierda: superación del dominio oligárquico y de las economías sustentadas en la producción agropecuaria o minera de exportación; por consiguiente, reforma agraria, impulso de la industrialización, de la urbanización, modernización de los aparatos de Estado (incluidos los educativos, por tanto, modernización o reforma de las universidades), lucha contra la pobreza, crecimiento de la «clase media». Las diferencias tenían que ver, sin embargo, con cuestiones de fondo: la dirección de los procesos (qué clases o grupos sociales debían dirigir los cambios), la vinculación con los centros del sistema mundial (especialmente, con los Estados Unidos), el carácter democrático (por tanto, qué clase de democracia: «liberal» o «popular») o dictatorial de los regímenes encargados de la modernización. Estas diferencias incidían desde luego en las distintas concepciones de reforma universitaria, aunque en todas ellas es fácilmente detectable su articulación con las ideas modernas de progreso, de dominio humano sobre la naturaleza, de crecimiento económico como condición del desarrollo social. Había que reformar o modernizar la universidad, para lo que se postulaban diferentes proyectos que tenían sin embargo un fundamento ideológico común: el desarrollo económico y social sustentado en el progreso científico y tecnológico. Hay que reconocer que Malo se situaba, con respecto a sus contemporáneos, un paso más allá, puesto que al menos introducía en el debate un cuestionamiento sobre la racionalidad científica y técnica. No obstante, lo que en los hechos marcó la efectiva transformación de la universidad provino de las tensiones entre las fuerzas sociales reales: 1) la «masificación», es decir, el crecimiento vertiginoso de la matrícula, en el cual inciden las presiones de sectores que veían su posibilidad de ascenso e incorporación social a través de la educación: nuevos grupos sociales urbanos, provenientes de la migración interna; adultos, especialmente mujeres, que habían visto interrumpidas sus expectativas por la urgencia de incorporarse al trabajo o por falta de recursos económicos o por la maternidad y la crianza de los hijos; 2) la diversificación de las instituciones de educación superior: universidades, politécnicas; universidades localizadas en las grandes ciudades -Quito, Guayaquil-, en ciudades de tradición universitaria —Cuenca, Loja-, o en ciudades de provincia de mediana dimensión; universidades públicas y privadas, confesionales, militares, de posgrado en ciencias sociales; 3) la creación de nuevas profesiones como resultado del desarrollo técnico, y 
4) la creciente participación del sector privado en la educación superior. Esta fue la «segunda reforma universitaria» realmente existente. El imprevisto y rápido crecimiento de la educación superior, sobre todo en la década de los años 70 del siglo pasado, vinculado a la escasez de recursos (tanto de profesores e investigadores como de recursos económicos), habría derivado, según se suele considerar, en la pérdida de calidad, es decir, en carencias en la formación profesional, en bajas tasas de eficiencia, en los resultados relativamente pobres de la investigación universitaria. De ahí que desde la década de los 80 se emprenda en América Latina la «tercera reforma» (como la ha denominado Claudio Rama) que tiene por divisa el «mejoramiento de la calidad», y que parte del reconocimiento de la diversidad de la educación superior, de la creciente privatización y del crecimiento de la población estudiantil como consecuencia de la «segunda reforma» ${ }^{3}$. No es una mera coincidencia que en América Latina se haya iniciado esta «tercera reforma» en la misma época en que comenzaba en Europa el llamado Proceso de Bolonia. Como no lo es tampoco que paralelamente se iniciara la crítica de la universidad tecnocrática (Lyotard, Derrida, S. Weber, Virilio o Nussbaum, para ilustrar ese esfuerzo crítico con el nombre de unos cuantos filósofos que, desde diferentes orientaciones, han intervenido sobre la cuestión en estas décadas), que ha implicado la crítica o la deconstrucción de las ideas modernas de universidad que provienen de la llustración, del Idealismo alemán y el Romanticismo de fines del siglo XVIII e inicios del siglo XIX, es decir, de las ideas que dirigieron la institución de la «universidad napoleónica» (llustración) o de la «humboldtiana» (Idealismo, Romanticismo). Los efectos de estos dos modelos universitarios, como es conocido, se incorporaron dentro de las estructuras tradicionales en las universidades anglo-

3 Como es sabido, la primera reforma, la de Córdoba, tuvo un contenido ilustrado y liberal. Su núcleo es la autonomía académica respecto del gobierno y otras instituciones estatales, autonomía que a su vez sustenta la libertad de cátedra vinculada con las libertades de pensamiento, de expresión, de prensa; sin esas libertades no habría posibilidad de progreso del conocimiento, de las ciencias. El cogobierno, la participación de profesores y estudiantes en el gobierno de la universidad, de alguna manera se relaciona con la «mayoría de edad» en la concepción kantiana y a la vez con la concepción liberal del ciudadano (que a la sazón tomaba en cuenta tanto la mayoría de edad como el nivel de ilustración alcanzado a través de la escolaridad). Sin embargo, para el filósofo Mayz Vallenilla, que llegó a ser rector-fundador de la Universidad Simón Bolívar de Venezuela, la autonomía universitaria provenía más bien de la organización hispánica premoderna de los gobiernos de las ciudades, los cabildos, lo que a él le llevaba a criticar la pretensión de república soberana que, desde su punto de vista, tenían las instituciones universitarias. 
sajonas (Reino Unido y Estados Unidos)4. En Hispanoamérica, los dos modelos universitarios, el «humboldtiano» y el «napoleónico» se injertaron tardía y parcialmente en una tradición que venía de la época colonial.

Más allá de la necesaria observación de la incoherencia que implica reducir la diversidad de instituciones de educación superior que existen en el país, con distintos propósitos, a una supuesta unidad, la crítica de lo que se ha denominado Universidad ecuatoriana tendría que interrogarse sobre dos aspectos que se entrelazan: la misión, esto es, las funciones sociales que se han asignado a la universidad, y la supuesta condición nacional de la institución universitaria. Ha sido común en los debates universitarios remitirse en América Latina, durante decenios, al ensayo Misión de la universidad de Ortega y Gasset (1930), escrito a partir de una conferencia pronunciada unos meses antes de la proclamación de la Segunda República Española, pero que puede considerarse como el manifiesto orteguiano de lo que debería ser la universidad republicana. En las legislaciones o declaraciones de principios de las instituciones universitarias hispanoamericanas se ha reiterado por decenios las tres funciones que componen la «misión de la universidad»: «l. Transmisión de la cultura._ II. Enseñanza de profesiones. - III. Investigación científica y educación de nuevos hombres de ciencia.» Se suele incluir en ocasiones una cuarta función, en rigor incluida en las dos primeras: la «extensión universitaria» (transformada luego en el Ecuador en «vinculación con la colectividad o con el medio externo»). Ortega tenía presentes tanto la concepción humboldtiana de universidad, pues al paso dirige una crítica a la universidad alemana de su tiempo, como el debate que había surgido, ya desde del siglo XIX, en torno a la primacía que debían tener o bien la enseñanza técnica, profesional, o bien la investigación y el progreso del conocimiento, debate que está relacionado con los dos modelos de universidad a la que nos hemos referido más arriba.

$4 \quad$ Posteriormente el sistema estadounidense de educación superior, en su evolución, ha llegado a ofrecer un abanico que tiene, en un extremo, los «colleges» (que son o institutos de educación técnica o una suerte de escuelas preparatorias pre-profesionales), y en otro, las grandes universidades de investigación, que son corporaciones universitarias ligadas a otras corporaciones financieras o de producción de alta tecnología. Max Weber advirtió a inicios del siglo pasado la condición empresarial que caracterizaba a las universidades estadounidenses; esa condición de empresa ha evolucionado, sobre todo en las grandes universidades de investigación, hasta adquirir la configuración de una gran corporación. 
¿Qué implica la «transmisión de la cultura», que Ortega coloca como función prioritaria sobre las otras dos, la enseñanza y la investigación? Es evidente que este postulado trae consigo una impronta a la vez ilustrada e idealista, a la vez que deja en el aire una serie de problemas relacionados con la cultura, con la tradición y sus rupturas (teniendo en cuenta que la tradición de la modernidad son las rupturas, según sostenía Octavio Paz), con las diversidades culturales que se encuentran, se confrontan y se modifican entre ellas, con las diferencias entre alta cultura y culturas populares... hasta llegar a las diferencias sexuales, de género, de edad, etcétera, de nuestros días. Sin embargo, hacia 1810, cuando en Hispanoamérica se iniciaba la Revolución de Independencia, y, por consiguiente, cuando se iniciaba la constitución de las naciones hispanoamericanas como «comunidades imaginadas» (Benedict Anderson), en medio de las guerras napoleónicas en que intervenían Prusia y los restantes Estados alemanes, Humboldt postulaba que en estricto rigor la principal función de la universidad (nosotros podríamos decir: de la universidad moderna) era la formación o la construcción (Bildung) de la cultura del Estado nacional (en su caso: Prusia, y en perspectiva, Alemania). La universidad «ecuatoriana» ha sido ciertamente un lugar privilegiado de la formación-construcción de la «cultura nacional» de Estado, por consiguiente, lugar en que se «sintetizan» o se incluyen en una unidad superadora las diferentes modalidades culturales, las diversas formas de la vida cotidiana, en una articulación histórica y antropológica que se pretende unitaria, o cuando menos convergente, en la formación de la «nación», del «pueblo ecuatoriano», o de «lo ecuatoriano» sin más: en otras palabras, su propósito es producir y reproducir ideológicamente la figura de la «identidad nacional». La crítica de esta concepción de la «cultura nacional», y consiguientemente de la imagen de «nación» que contribuye a crear, se viene realizando desde hace un buen tiempo, desde mediados del siglo pasado, y sobre todo en las universidades, o en algunas de ellas. No obstante, la idea de «cultura nacional», asociada a las nociones (ideológicas) de identidad cultural e identidad nacional, y hasta de soberanía cultural es persistente en los discursos políticos, en los textos legales y en las ideologías culturales, tanto conservadoras como progresistas o populistas ${ }^{5}$. Los Estados nacionales surgen en vínculo con las

5 La Ley Orgánica de Cultura, promulgada en diciembre de 2016, reitera los fundamentos de esta ideología, aunque intenta encubrirla con una lamentable retórica, mediante la cual sus creadores se esfuerzan por juntar la «identidad» con la «diversidad». Pese a los intentos de encubrimiento, su objetivo se declara explícitamente: «ldentidad nacional. Se construye 
culturas nacionales y tienen que promoverlas como uno de sus fundamentos. La universidad moderna contiene en sí misma una tensión que emerge de la contradicción entre su función estatal y social, en sí conservadora, esto es, la formación, construcción y reproducción de la cultura nacional, que tiende a la identidad, y la incidencia de las formas culturales que provienen tanto del mundo exterior a las fronteras nacionales (digamos, hoy día, de la mundialización o globalización) como de la multifacética diversidad interna de los Estados, muchas de las cuales son potencialmente irruptoras y podrían impulsar la transgresión de la cultura nacional de Estado. La indudable crisis de la noción (ideológica) de cultura nacional, incluso si se intenta encubrirla con la incorporación de la diversidad de identidades culturales, que sin embargo se inscribirían en una historia que confluiría en un presente que las unifica, está relacionada con la crisis de los Estados nacionales que caracteriza a nuestra época, cuando la globalización ha modificado el orden mundial, que hoy día se articula a través de relaciones geopolíticas regidas por relaciones de poder entre grandes conglomerados estatales y corporativos, y por regulaciones y normas que delimitan las pretensiones soberanistas de los Estados nacionales. Vivimos en una época en que los Estados nacionales formados en los siglos XIX y XX transitan hacia otras formas políticas. A la vez, la unidad del Estado nacional se mantiene en tensión con las diferencias étnicas o religiosas internas, que generan irrupciones y en ocasiones fracturas nacionalistas ${ }^{6}$. Más aún, hoy se advierten varios signos de crisis de algunos Estados cuyas formas de dominio provienen de o se articulan con estructuras mafiosas o clericales, en el caso de los llamados fundamentalismos, esto es, poderes que trastocan por completo fronteras, que generan articulaciones entre política, economía y negocios legales e ilegales impredecibles. En consecuencia, debemos preguntarnos sobre la actualidad de la divisa orteguiana «transmisión de la cultura», no porque ya no hubiese una cultura que trasmitir, o menos aún porque la universidad hubiese dejado

y afirma a través del conjunto de interrelaciones culturales e históricas que promueven la unidad nacional y la cohesión social a partir del reconocimiento de la diversidad».

6 El primer artículo de la Constitución de la República del Ecuador ya pone en evidencia esta crisis inherente al Estado nacional ecuatoriano, cuando a más de caracterizarlo como «soberano, independiente, unitario, intercultural», lo declara «plurinacional», aunque en el contexto constitucional no aparezca ninguna consecuencia de tal declaración; tampoco en el contexto legal derivado de la Constitución, y aún menos en la organización del Estado. 
de ser un lugar de reproducción de las formas culturales, sino por las sustanciales modificaciones que estas adquieren en la mundialización y la mutación de lo local o lo nacional.

Se suele entender por «cultura» un entramado de creencias compartidas, lenguajes, valores morales, actitudes colectivas o hábitos, que se constituyen en prejuicios o presupuestos a partir de los cuales se aprecian las situaciones, se juzgan entre sí los individuos que interactúan, o se evalúa lo que se tiene a mano para tomar decisiones. La universidad también en este sentido es un lugar de producción y transmisión de la cultura. En el Ecuador, la universidad pública fue, en su momento, la portadora de los valores del laicismo liberal; pero luego surgieron las universidades católicas para contraponer a ese laicismo una concepción confesional, en primera instancia conservadora, así como las escuelas politécnicas en las que ha primado, como es obvio, una ideología tecnocrática, vinculada con el desarrollismo. Sin embargo, ya desde finales del siglo pasado la hegemonía de la ideología tecnocrática es evidente, con sus consiguientes nociones de eficiencia, eficacia, emprendimiento, y en conexión con estas, la noción de meritocracia, con la que se pretende desconocer la brutal desigualdad social que ubica a los individuos en condiciones sustancialmente diferentes desde su nacimiento, incluso desde su concepción, distinción que profundiza el sistema educativo (Bourdieu). La ideología tecnocrática se centra en la formación del «capital humano» y en la innovación incesante de las tecnologías y, a través de estas, en la profundización del control y la administración de las poblaciones, de la naturaleza y sus recursos bajo la hegemonía de las grandes corporaciones. Frente a la hegemonía de la ideología tecnocrática, los sistemas de valores, creencias y hábitos provenientes del humanismo e incluso del liberalismo (político) han entrado en crisis desde hace tiempo (1968 es en este sentido una fecha cargada de significación). Frente al viejo humanismo y su deriva en varias formas de «compromiso» existencial, confesional o político, prevalecen el individualismo y el cinismo (no el de Diógenes, desde luego). Pese a ello, todavía en las universidades ecuatorianas se escuchan los cánticos nostálgicos de sectores conservadores que provienen tanto de cierta izquierda anclada en el nacionalismo, como de cierto liberalismo (radical, en el sentido que tiene el término en el ambiente académico estadounidense) que se expresa en el multiculturalismo o en el énfasis extremadamente particularista 
que se otorga a distintas «identidades» (étnicas, sexuales, de género, etcétera). Sin embargo, hay otros valores, otras formas de cohesión grupal, de solidaridad, que si bien no son los que predominan en el ámbito universitario, irrumpen cuando menos de manera parcial, localizada o puntual, contra la cultura hegemónica en las universidades. Una de estas formas suele emerger en la cooperación entre profesores y estudiantes, cuando se logra romper con las jerarquías autoritarias asociadas a la detentación del saber. ¿Podrá emerger desde ellas la crítica que requiere la universidad tecnocrática contemporánea?

La enseñanza técnica, profesional, está marcada por la ideología tecnocrática, aunque esta se encubra ideológicamente, y a pesar de los cuestionamientos éticos o políticos de índole liberal (por caso, la apelación a los derechos humanos) o humanitaria (la solidaridad con los pobres, con los más vulnerables). Para una posición crítica, habría que preguntarse por las concepciones de salud y enfermedad, de ciudad, de vivienda, espacio público, territorio, seguridad, legalidad, legitimidad, derechos y justicia, información, vida cotidiana humana y vida en general, que prevalecen en las enseñanzas, más allá de los discursos del humanitarismo o los derechos humanos o el «buen vivir», que pueden recitarse sin problema en las universidades tecnocráticas. Algo semejante se puede decir a propósito de la investigación: ¿hasta qué punto el avance científico ha quedado subsumido en la producción de tecnologías? ¿Hasta qué punto estas están subordinadas a los intereses de las grandes corporaciones, a los intereses militaristas y las guerras, al control y administración de las poblaciones? ¿Qué es lo que se puede investigar en nuestras universidades, las ecuatorianas, sin ilusión alguna, y con qué fines? Los estudios humanísticos han sido más bien marginales en nuestras universidades. Salvo contadísimas excepciones, no ha habido en ellas, durante décadas, lugar para la formación de filósofos, historiadores, críticos literarios, lingüísticas, etnólogos. Hoy esos estudios tienden a la extinción o se los encierra en facultades (especialmente en las de pedagogía) que nada tienen que ver con la formación de investigadores en estos campos del saber, o directamente se los suprime. Esta marginalidad es en gran medida consecuencia del dominio de la concepción tecnocrática dentro de la universidad. Sin embargo, algo parecido ocurre en el ámbito de las ciencias: apenas si hay alguna universidad o politécnica donde se formen investigadores en biología, matemáticas, física, química. Habría que indagar si, más allá de las apariencias, se forman investigadores en las ciencias sociales, y no solo especialistas en el manejo 
de técnicas de administración (gestión, planificación, control) social o empresarial.

Sin embargo, hay algo más complejo hacia lo que debemos aproximarnos. La actual crisis de las Humanidades no es solo un efecto de la ideología tecnocrática hegemónica; es una crisis que surge de la radical transformación que se opera en la condición humana como consecuencia de la revolución tecnológica posterior a la Segunda Guerra Mundial. Es una crisis derivada de los efectos de esta revolución tecnológica en la vida de los seres humanos y en su configuración como sujetos, en los efectos sobre las otras formas de vida e incluso sobre la estructura geológica del planeta. Aquí solo podemos enunciar algunas de las cuestiones que atañen a la «condición humana», si es posible utilizar aún esta terminología, y a la vida en la Tierra. Son cuestiones que han recibido una atención muy limitada dentro de las universidades y dentro de la «opinión pública»; se diría incluso que están lejos de ser consideradas con el rigor y la atención que merecen, pese a la gravedad de lo que acontece en la Tierra. La cresta más visible de esta gravedad es lo que conocemos como «cambio climático», cuyos efectos catastróficos se evidencian día a día: sequías e inundaciones, incendios forestales, deshielo de glaciares y de los polos... Causa estupor, y con sobrada razón, que el presidente del Estado más poderoso del mundo declare que saca a su país del Acuerdo de París de 2015; sin embargo, no nos preguntamos si hay avances reales que sean consecuencia de ese Acuerdo y si su alcance será suficiente para conseguir la meta, ya por sí misma grave, de evitar que el incremento de la temperatura media de la Tierra sobrepase los $2^{\circ} \mathrm{C}$ en el año 2100, tomando como referencia la temperatura media calculada para 1880 (el aumento hasta 2015 ya era de $0.8^{\circ} \mathrm{C}$ ). Sabemos que este calentamiento global está vinculado a la emisión de dióxido de carbono y otros compuestos que inciden en lo que se ha denominado «efecto invernadero»; por consiguiente, es el efecto de una compleja red de actividades humanas, comenzando por el uso de combustibles fósiles (petróleo, carbón), en la que actualmente estamos involucrados todos los seres humanos. La actividad humana incide negativamente en otros ámbitos: contaminación del aire, de los suelos, de ríos, lagunas, mares, destrucción de bosques y otros ecosistemas; en una palabra, devastación de la Tierra. En esta época, la nuestra, está en curso la sexta gran extinción de especies vivas en el planeta; esta extinción no es solo un acontecimiento catastrófico dentro de la «historia natural», sino que en ella 
tiene una directa responsabilidad la especie humana7 , sobre todo en el curso de su historia moderna (determinada, es cierto, por el capitalismo, pero igualmente por las formas de socialismo, igualmente supeditadas al productivismo). Asistimos a una catástrofe en cuyo curso vemos desaparecer las especies más cercanas a la humana, o vemos que sus poblaciones se reducen brutalmente, y apenas si se conservan en reservas para los estudios genéticos o etológicos. La incidencia de la actividad humana, especialmente en la Modernidad, y con una fuerza inusitada luego de la Segunda Guerra Mundial, ha provocado una transformación radical de la Tierra, a tal punto que hoy se considera que cabe hablar de una ruptura geológica, de una nueva era, el Antropoceno, posterior al Holoceno, que es el período donde se realizó la mayor parte de la existencia de nuestra especie.

Cuando parecía que se había alcanzado finalmente el «dominio del hombre sobre la naturaleza» y se suponía que se superaba la escasez, la actividad humana ha acabado por producir un vasto ecocidio, que es el término cabal para nombrar la devastación en curso, que es la consecuencia de las estrategias productivistas de uso intensivo de los «recursos naturales» (tanto de animales, vegetales y otras formas de vida, como de recursos minerales). No se debería tomar el ecocidio en un sentido apocalíptico, pues no se trata de una profecía; es un acontecimiento que viene sucediendo y que sabemos que continuará. No se trata tampoco del anuncio del fin irremediable de lo humano, aunque en el curso de la catástrofe a la que asistimos se revele también la vulnerabilidad de la especie humana y la posibilidad de su extinción o de su transmutación. Asimismo, se revela la diferencia entre poblaciones más vulnerables que otras, más amenazadas por las hambrunas, por los desastres vinculados con el cambio climático; y también con ello se revela la desmesura que ha adquirido la población humana con respecto a los recursos del planeta y los desequilibrios demográficos: grandes concentraciones en algunos países, megalópolis, migraciones, entre las que irán en aumento las ocasionadas por los desastres ambientales.

$7 \quad$ En la naturaleza, con independencia de la actividad humana, surgen nuevas especies a la vez que otras mutan o se extinguen. Para considerar la incidencia de la actividad humana sobre la sexta extinción se comparan las proyecciones de las tendencias naturales con los datos de la real extinción de especies en el curso de la época moderna y el presente (Ceballos et al., 2015). 
La posibilidad de extinción de la especie humana se combina, de modo contradictorio, con la posibilidad paralela de alargar la vida de los individuos humanos (lo que hasta cierto punto puede vincularse con un anhelo de inmortalidad, que en la esfera religiosa se manifestaba en las concepciones de resurrección o de reencarnación), lo que podría augurar la salida «salvadora» de la especie hacia algún exoplaneta, como ha sugerido Stephen Hawking. Esta prolongación de la vida humana depende de los avances científicos y tecnológicos, de la posibilidad de manipulación genética (no solo sobre humanos sino sobre el conjunto de los seres vivos), de las técnicas médicas, de las prótesis. Los seres humanos dependemos siempre de los contextos artificiales que se configuran históricamente, de las herramientas, de las máquinas, de los objetos prácticos que consumimos para nuestra reproducción como individuos y como especie. Dependemos de los contextos artificiales heredados y de las modificaciones en el curso de la existencia. Lo peculiar de lo humano está en el lenguaje verbal, en la capacidad para producir artefactos, en la capacidad para transformar las condiciones de la reproducción social (de la especie, de la forma social y de los individuos). Con ello se configuran distintas modalidades de «paisaje», de entornos habitables, de símbolos; los individuos nacemos en contextos artificiales, culturales. Lo peculiar del presente es que, como humanidad, hemos llegado a una configuración civilizatoria mundializada, en la que se despliega un desarrollo tecnológico de constante aceleración. En ese contexto, la genética y las tecnologías asociadas con ella, han situado al ser humano en una proximidad de lo animal, tanto por el parentesco genético como por la posibilidad de manipulación genética, que sacude la arrogancia mantenida por milenios, enlazada con las creencias religiosas o filosóficas, que establecían un corte radical entre lo animal y lo humano. Por otra parte, el desarrollo tecnológico de artefactos (las prótesis, entre las que habría que contar el sinnúmero de aparatos que requerimos para comunicarlos, movilizarnos, interactuar, o los robots, que cada vez más aceleradamente sustituyen a los trabajadores humanos: finalmente, el cyborg) han cambiado la idea de «ser humano» o ideas subsidiarias de esta, como la de «inteligencia». ¿Qué es «ser humano»?, es una pregunta insólita que crea en nosotros perplejidad. Esta perplejidad, lo insólito de esta pregunta, produce una conmoción en las configuraciones del saber contemporáneo; es una perplejidad que pareciera emerger del fondo del campo de percepción del mundo, de ese fondo 
siempre oscuro a partir del que se configuran las concepciones del mundo y del que surgen los conocimientos y la posibilidad de comprendernos a nosotros-mismos. ¿Qué es ser humano en una situación en que está en riesgo la supervivencia de la especie como consecuencia de la catástrofe de gigantescas proporciones ocasionada por la actividad humana, y en que las tecnologías contemporáneas están transformando radicalmente las condiciones de lo humano? A tal pregunta se suceden otras: ¿Qué es ser inteligente? ¿Qué es ser trabajador o qué es ser intelectual? ¿Qué es conocer? ¿Sabiduría?...

Perplejidad ante la «condición humana» contemporánea, la de la extinción posible de la especie o de su posible transmutación a través de las tecnologías; perplejidad ante la catástrofe natural en curso, que exigiría por parte del conjunto de la humanidad una serie compleja de acciones que suponen concertaciones políticas y desarrollos tecnológicos ya no supeditados a la lógica capitalista de obtención de plusvalía (ganancia, renta tecnológica); perplejidad ante los desequilibrios demográficos. Perplejidad que se junta al abandono de las pretensiones de los determinismos, de la supuesta capacidad para calcular los resultados de las acciones humanas; perplejidad, por tanto, vinculada con el paso fundamental del determinismo derivado de la mecánica clásica al principio de incertidumbre... Perplejidad ante la crisis de las formas políticas, en especial la crisis de la democracia... ¿Cómo concebir el presente, la actualidad, en esa condición de perplejidad? ¿Qué ética cabría postular, qué se puede esperar para los seres humanos actuales y para los que están por venir? ¿A qué libertad, a qué comunidad se podría aspirar?

Volvamos a la cuestión de la universidad, esto es, de lo que podríamos proponer para las universidades ecuatorianas hoy en día. Se tiene que derogar el autoritarismo burocrático impuesto mediante la ley de 2010 y por la legislación dictada por los organismos gubernamentales que aquella creó; sin embargo, hay que tener presente que esa acción necesaria y urgente no modificará en lo sustancial la hegemonía de la ideología tecnocrática, aunque modifique las prácticas autoritarias. La ideología tecnocrática proviene de configuraciones de la organización actual de los poderes y los saberes, de su carácter a la vez global, nacional-estatal y local. La hegemonía ideológica, por lo demás, no solo actúa de manera autoritaria, sino también de modo democrático; se puede decir más bien que requiere del autoritarismo ahí donde el poder político manifiesta su debilidad en el control del «pueblo». 
La democracia liberal posibilita ciertamente la libertad de investigación, de expresión o de cátedra, que permiten la crítica limitada de la ideología tecnocrática dominante, sobre todo desde formas liberales-radicales (es decir, que tiene raíces en el liberalismo), vinculadas con los derechos humanos o con el humanitarismo. Pero, ¿qué pasa si se pone en cuestión el conjunto de los presupuestos democrático-liberales? Tal puesta en cuestión en el plano teórico no es sino la puesta en correspondencia de la teoría con la realidad, pues es más que evidente la crisis de la democracia liberal, representativa, en el mundo actual. La democracia liberal es ante todo una forma política occidental, europea y americana; ¿qué podemos decir de tal democracia sometida a los simulacros, la mentira repetida en la propaganda, hoy llamada «postverdad», la corrupción? Ante la crisis de tal democracia, que viene de lejos, reaparece constantemente la invocación a la democracia directa, plebiscitaria, en nombre de la soberanía popular, y ya se sabe de sobra cómo tal democracia directa finalmente acaba en la dictadura autoritaria de los caudillos. ¿Cuáles podrían ser las formas democráticas, republicanas, a las que todavía podríamos aspirar? ¿Pueden surgir estas de las posibilidades abiertas por las nuevas tecnologías de comunicación e información? Estas, como observamos, son a la vez vehículo del simulacro y la mentira, o vehículo de formas de asociación democráticas (más bien circunscritas en sus propósitos, puntuales, fugaces).

No obstante, si no existiese dentro de las instituciones que se llaman «universidad» un mínimo de condiciones para la crítica, para la puesta en cuestión de los sistemas de conocimiento o de los órdenes de verdad existentes; si no existiese un mínimo de condiciones para el examen crítico o deconstructivo de las formas culturales o ideológicas, los valores, los hábitos, las creencias, en suma, los discursos; si no existiese un mínimo de condiciones que aseguren la posibilidad de disentir, de exponer argumentos que vayan contra las corrientes hegemónicas, no habría en rigor «universidad». En este sentido, cabe aún hoy reivindicar que la universidad es «sede de la razón», o mejor aún, del pensamiento. Hernán Malo señalaba que incluso si las instituciones así llamadas «universidades» dejasen de existir, si dejasen por tanto de ser espacios para el despliegue libre de la razón o del pensamiento, siempre habría lugares o modalidades de asociación en las que se seguirían formulando las preguntas que deben ser planteadas y se seguiría dialogando críticamente. Lo cual, en efecto, sucede. En este sentido, la autonomía universitaria, tal como fue concebida por el liberalismo, da paso a la 
cooperación amplia, tendencialmente general, esto es, abierta a la participación de cualquier ser humano (o posthumano), lo que pone en crisis la concepción meritocrática y los residuos aristocráticos, y por consiguiente de arribismo social, asociados con el prestigio del título académico o profesional (prestigio que se ha tornado aberrante entre nosotros, los ecuatorianos). Hoy día la cooperación está subordinada a las necesidades de acuerdo entre corporaciones (transnacionales) y poderes políticos, pero a la vez hay líneas de fuga, posiciones de frontera, en que son posibles otras formas de cooperación. Una universidad que renuncie al claustro, a los muros (no solo a las paredes sino a los muros mentales) es posible y necesaria. Esta, una universidad abierta, donde sea posible contar con las condiciones mínimas para la libertad del pensamiento, para la crítica o la deconstrucción, para la disensión, es el lugar necesario, el ámbito de extrema exigencia con nosotros-mismos, que aspiramos a tener la autonomía, es decir, la libertad y madurez necesarias que nos permitan inquirir sobre «nosotros-mismos» en esta época de perplejidad. 


\section{BIBLIOGRAFÍA:}

Braidotti, Rosa (2105). Lo posthumano. Barcelona, España: Gedisa.

Carvajal, I. (2016). Universidad. Sentido y crítica. Quito, Ecuador: PUCE.

(2016). «La PUCE y los desafíos hacia el futuro», en: PUCE. Pensamiento, ciencia, sociedad. Quito, Ecuador: PUCE.

Ceballos, G.; P. R. Ehrlich, A. D. Barnosky, A. García, R. M. Pringle, T. M. Palmer (2015) Accelerated modern human-induced species losses: Entering the sixth mass extinction. Documento recuperado el 20.06.2016 de: http://advances.sciencemag.org/content/1/5/ e1400253.full-text.pdf+html

Clark, W. C., P. J. Crutzen, H. J. Schellnhuber (2005). Science for Global Sustainability: Toward a New Paradigm. Harvard University, John F. Kennedy School of Government. Documento recuperado el 15.02.2016 de https://www.hks.harvard.edu/content/download/69374/1250306/version/1/file/120.pdf

Cohen, Tom; Claire Colebrook y J. Hillis Miller (2016). Twilight of the Antropocene Idols, London, Open Humanities Press. Recuperado el 15.02.2016 de http://www.openhumanitiespress.org/books/titles/twilight-of-the-anthropocene-idols/

Derrida, J. (2001). La Universidad sin condición. (C. d. Peretti, Trad.) Madrid, España: Trotta

Foucault, M. (1984) Qu'est-ce que le Lumièrs? Documento recuperado el 10.06.2017 de http://1 libertaire.free.fr/Foucault17.html. Trad. en español: Sobre la Ilustración, Madrid, España; Tecnos (2003).

Herbrechter, S. (2013). Rosi Braidotti (2013) The Posthuman, en Culture Machine, documento recuperado el 10.08.2016 de http://www.culturemachine.net/index.php/cm/ article/viewfile/495/516

Joignot, F. (2016). Robotisation générale, Le Monde, Culture \& Idées, 2-3-4.01.2016, pp. 4-5

Lyotard, J. F. (1994). La condición postmoderna, informe sobre el saber. Madrid, España: Cátedra.

Malo González, H. (1976). «Universidad, sede de la Razón». Revista de la Universidad Católica del Ecuador (13). Quito, Ecuador: PUCE. 
Nussbaum, M. C. (2010). Sin fines de lucro. Por qué la democracia necesita de las humanidades. Buenos Aires, Argentina: Katz

Ortega y Gasset, J. (2007). Misión de la Universidad. Madrid, España: Biblioteca Nueva.

Villavicencio, A. (2013). ¿Hacia dónde va el proyecto universitario de la revolución ciudadana? Quito, Ecuador: Universidad Andina Simón Bolívar.

(2013). De la universidad funcional a la universidad de la razón. Quito, Ecuador: Universidad Andina Simón Bolívar.

(2016). Calidad, excelencia y evaluación en la universidad ecuatoriana. Quito, Ecuador: Paradiso - UASB.

Virilio, P. (2007). L'Université du désastre. Paris, Francia: Galilée. 